\title{
R-CHOP/R-DHAP Regimen
}

National Cancer Institute

\section{Source}

National Cancer Institute. R-CHOP/R-DHAP Regimen. NCI Thesaurus. Code C140734.

A regimen that alternates rituximab, cyclophosphamide, doxorubicin, vincristine and prednisone (R-CHOP) with rituximab, dexamethasone, high-dose cytarabine and cisplatin (R-DHAP), and can be used in the treatment of mantle cell lymphoma. 